\title{
Autoresonant-spectrometric determination of the residual gas composition in the ALPHA experiment apparatus
}

\author{
C. Amole, ${ }^{1}$ M. D. Ashkezari, ${ }^{2}$ M. Baquero-Ruiz, ${ }^{3}$ W. Bertsche, ${ }^{4,5}$ E. Butler, ${ }^{6, a)}$ A. Capra, ${ }^{1}$ \\ C. L. Cesar, ${ }^{7}$ S. Chapman, ${ }^{3}$ M. Charlton, ${ }^{4}$ S. Eriksson, ${ }^{4}$ J. Fajans, ${ }^{3,8}$ T. Friesen, ${ }^{9}$ M. C. \\ Fujiwara, ${ }^{9,10}$ D. R. Gill, ${ }^{10}$ A. Gutierrez, ${ }^{11}$ J. S. Hangst, ${ }^{12}$ W. N. Hardy,,${ }^{11,13}$ M. E. Hayden, ${ }^{2}$ \\ C. A. Isaac, ${ }^{4}$ S. Jonsell, ${ }^{14}$ L. Kurchaninov, ${ }^{10}$ A. Little, ${ }^{3}$ N. Madsen, ${ }^{4}$ J. T. K. McKenna, ${ }^{15}$ \\ S. Menary, ${ }^{1}$ S. C. Napolii, ${ }^{4}$ P. Nolan,${ }^{15}$ K. Olchanski, ${ }^{10}$ A. Olin,,${ }^{10,16}$ A. Povilus, ${ }^{3}$ P. Pusa,${ }^{15}$ \\ C. Ø. Rasmussen, ${ }^{12}$ F. Robicheaux,${ }^{17}$ E. Sarid, ${ }^{18}$ D. M. Silveira, ${ }^{7}$ S. Stracka,${ }^{10}$ C. So,${ }^{3}$ \\ R. I. Thompson, ${ }^{9}$ M. Turner, ${ }^{3}$ D. P. van der Werf, ${ }^{4}$ J. S. Wurtele, ${ }^{3,8}$ and A. Zhmoginov ${ }^{3,8}$ \\ (ALPHA Collaboration) \\ ${ }^{1}$ Department of Physics and Astronomy, York University, Toronto, Ontario M3J 1P3, Canada \\ ${ }^{2}$ Department of Physics, Simon Fraser University, Burnaby, British Columbia V5A 1S6, Canada \\ ${ }^{3}$ Department of Physics, University of California, Berkeley, California 94720-7300, USA \\ ${ }^{4}$ Department of Physics, Swansea University, Swansea SA2 8PP, United Kingdom \\ ${ }^{5}$ School of Physics and Astronomy, University of Manchester, Manchester M13 9PL, United Kingdom \\ and The Cockcroft Institute, Daresbury Laboratory, Warrington WA4 4AD, United Kingdom \\ ${ }^{6}$ Physics Department, CERN, CH-1211 Geneva 23, Switzerland \\ ${ }^{7}$ Instituto de Física, Universidade Federal do Rio de Janeiro, Rio de Janeiro 21941-972, Brazil \\ ${ }^{8}$ Lawrence Berkeley National Laboratory, Berkeley, California 94720, USA \\ ${ }^{9}$ Department of Physics and Astronomy, University of Calgary, Calgary, Alberta T2N 1N4, Canada \\ ${ }^{10}$ TRIUMF, 4004 Wesbrook Mall, Vancouver, British Columbia V6T 2A3, Canada \\ ${ }^{11}$ Department of Physics and Astronomy, University of British Columbia, Vancouver, \\ British Columbia V6T 1Z1, Canada \\ ${ }^{12}$ Department of Physics and Astronomy, Aarhus University, DK-8000 Aarhus C, Denmark \\ ${ }^{13}$ Canadian Institute of Advanced Research, Toronto, Ontario M5G-1Z8, Canada \\ ${ }^{14}$ Department of Physics, Stockholm University, SE-10691 Stockholm, Sweden \\ ${ }^{15}$ Department of Physics, University of Liverpool, Liverpool L69 7ZE, United Kingdom \\ ${ }^{16}$ Department of Physics and Astronomy, University of Victoria, Victoria, British Columbia V8W 3P6, Canada \\ ${ }^{17}$ Department of Physics, Auburn University, Auburn, Alabama 36849-5311, USA \\ ${ }^{18}$ Department of Physics, NRCN-Nuclear Research Center Negev, Beer Sheva IL-84190, Israel
}

(Received 17 April 2013; accepted 30 May 2013; published online 25 June 2013)

\begin{abstract}
Knowledge of the residual gas composition in the ALPHA experiment apparatus is important in our studies of antihydrogen and nonneutral plasmas. A technique based on autoresonant ion extraction from an electrostatic potential well has been developed that enables the study of the vacuum in our trap. Computer simulations allow an interpretation of our measurements and provide the residual gas composition under operating conditions typical of those used in experiments to produce, trap, and study antihydrogen. The methods developed may also be applicable in a range of atomic and molecular trap experiments where Penning-Malmberg traps are used and where access is limited. (C) 2013 AIP Publishing LLC. [http://dx.doi.org/10.1063/1.4811527]
\end{abstract}

\section{INTRODUCTION}

The residual gas in charged particle traps has important effects on the confined species. It may affect plasma expansion rates, ${ }^{1}$ allow chemical reactions, ${ }^{2}$ and, in the case of antimatter plasmas, promote annihilation of the particles. In devices intended to trap antihydrogen, ${ }^{3}$ it may also determine the lifetime of the trapped anti-atoms. Indeed, for antimatter plasmas, knowledge of the residual gas composition, together with the measurement of the annihilation rate and with suitable cross-section calculations, can be used to determine the residual gas density. ${ }^{4}$

For cryogenically cooled traps, composition measurements inside the trap itself are required. Determinations of

a) Present address: Centre for Cold Matter, Imperial College, London SW7 2BW, United Kingdom. the gas composition with conventional residual gas analyzers in room temperature regions of the apparatus are not useful because many species condense on the cryogenic trap surfaces, thereby changing the composition of the gas in the trap itself. For example, the ALPHA experiment, ${ }^{5}$ which studies antimatter at cryogenic temperatures, operates at an internal system temperature of $9 \mathrm{~K}$ and thus the only gases in thermal equilibrium with the wall that are not expected to have largely condensed are helium, hydrogen, and neon. ${ }^{6}$ There is, however, the possibility of a "wind" of other species, such as $\mathrm{N}_{2}$ and $\mathrm{O}_{2}$, flowing from warmer areas of the apparatus, including some areas at room temperature that are not closed off from the trap interior.

Because of limited access, strong magnetic fields, and cryogenic temperatures, it is not possible to implement conventional residual gas analysis (RGA) techniques in the ALPHA apparatus. Consequently, we have developed a 
technique that relies on autoresonant ion extraction from an electrostatic potential well. This method is related to the one used in a commercial autoresonant mass spectrometer ${ }^{7,8}$ and can be implemented in the standard ALPHA PenningMalmberg trap without modification to the trap geometry. It may also be applicable to other Penning trap devices where access is limited.

Autoresonance is a very general phenomenon occurring in many different dynamical systems ${ }^{9,11,12}$ that allows a weakly damped, driven, nonlinear oscillator to phase lock to its drive. For example, consider an initially quiescent oscillator with a soft non-linearity (an oscillation frequency that decreases with amplitude). If the oscillator is subject to a sweptfrequency drive that starts well above the oscillator's linear frequency and which sweeps downward through and below the linear frequency, then phase-locking will occur automatically, subject to some understood criteria. It has been shown that the drive amplitude must be proportional to the sweep rate raised to the $3 / 4$ power for phase locking to occur. ${ }^{9,10}$ The oscillator amplitude will then adjust itself to match the drive frequency and, consequently, can be controlled (and made very large) simply by decreasing the drive frequency. This autoresonant process will work even with a very weak drive. ${ }^{10}$ It will also occur when the oscillator is not initially quiescent, but then the phase locking condition is more complicated, and the oscillator can lock to subharmonics of the drive. ${ }^{13}$

The residual gas composition is measured by ionizing the residual gas with hot positrons (see Fig. 1), whereupon the positrons and ions are separated, with the latter placed in an anharmonic electrostatic potential well. Autoresonance is then used to selectively extract the ions from the well, where the drive frequency at which the ions escape depends on their mass-to-charge ratio. The escaping ions are collected with an MCP (Microchannel Plate) amplifier, and the amplified charge signal is compared to simulations of the process to determine the gas composition.
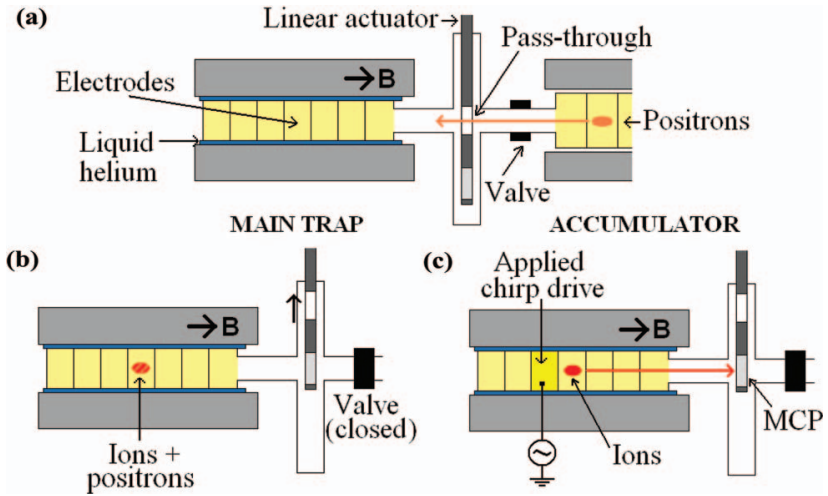

FIG. 1. The main ALPHA trap and positron accumulator are PenningMalmberg traps, where charged particles are confined radially by solenoidal magnetic fields, and longitudinally by electric fields produced with hollow cylindrical electrodes. Both traps are separated by a valve that is only opened for positron transfers. A linear actuator is also located between the traps that either allows positrons to pass through, or inserts an MCP for charge detection. (a) Configuration for positron transfers. (b) Configuration for charged particle manipulations. (c) Detection of autoresonantly extracted ions by charge amplification with the MCP

\section{THE ALPHA APPARATUS}

The ALPHA experiment, located at CERN's Antiproton Decelerator facility, has been designed and optimized to produce neutral antihydrogen atoms ${ }^{3,5}$ by carefully combining plasmas of antiprotons and positrons in a Penning-Malmberg trap kept at cryogenic temperatures and under ultra-high vacuum conditions (Fig. 1). The charged particles are confined radially by a homogeneous $1 \mathrm{~T}$ magnetic field produced by a superconducting solenoid. Axial confinement is achieved with electric fields produced with a set of over 30 electrodes whose electric potentials can be individually controlled. Once produced, neutral anti-atoms are trapped using a combination of octupole and magnetic mirror magnetostatic fields. For the work presented here, the neutral trap was de-energized at all times.

In a typical run, $\sim 10^{7}$ positrons are brought from a Surko-type positron accumulator ${ }^{14}$ into the main trap ${ }^{15}$ (Fig. 2(a)) with an energy of $\sim 80 \mathrm{eV}$, and trapped in an electrostatic potential well with a minimum near the center of

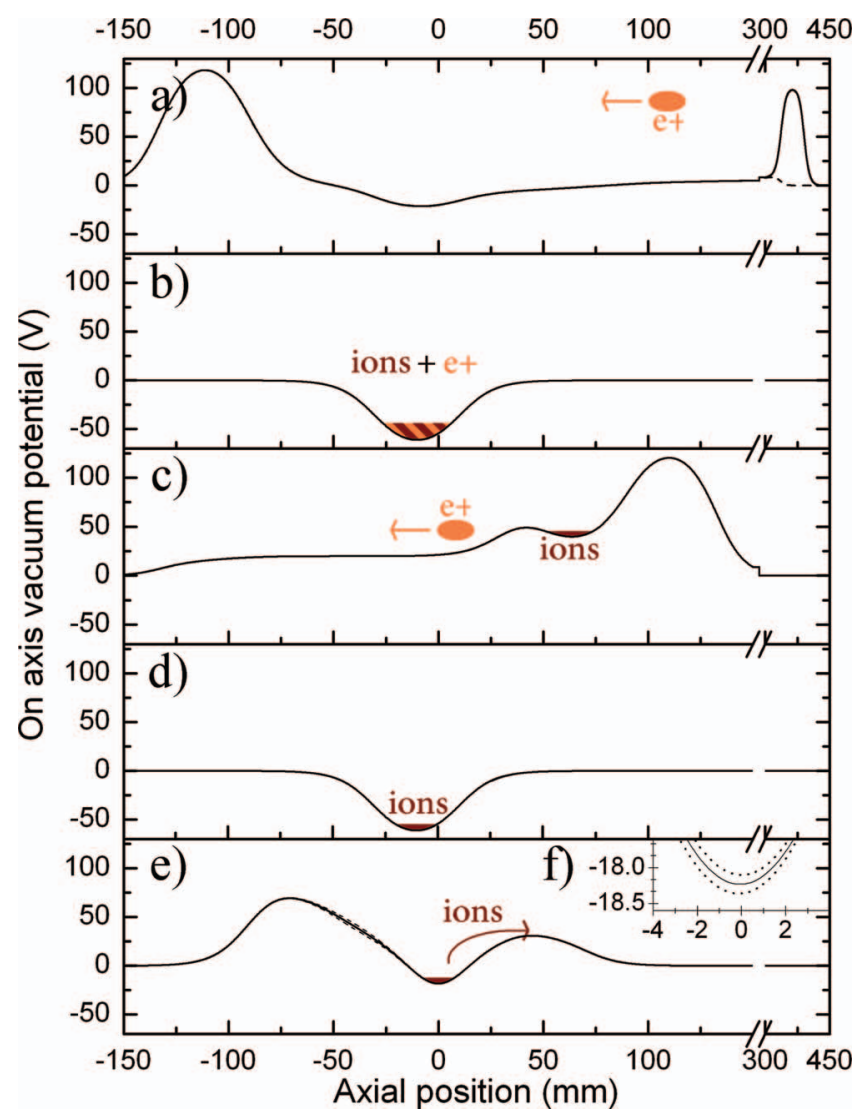

FIG. 2. Electrostatic potential manipulations during a typical run. (a) Positrons are brought into the main trap from the right, at a time when there is no potential wall at the axial position $\sim 400 \mathrm{~mm}$ (dashed line). As soon as they enter, the potential wall is erected (solid line) and positrons are confined and forced to bounce inside the experiment. Ions are formed through collisions of the positrons with the residual gas. (b) Ions and positrons cool down and accumulate at the bottom of the potential well, which is meanwhile modified to a more localized shape. (c) Positrons are then separated and discarded, and (d) ions are placed back in a deep well. (e) Finally, ions are placed at the bottom of an anharmonic well (solid line). A chirp is then applied that autoresonantly forces the ions to escape onto the MCP. The maximum electrostatic potential variations due to an applied $5 \mathrm{~V}$ chirp are shown as dashed lines. (f) Potential variations due to the chirp near the well minimum. 
the electrode stack. The confined positrons bounce in this initial electrostatic well with enough energy to ionize atoms or molecules of the residual gas through collisions. ${ }^{16-18}$ Ions with low enough energy are trapped in the well along with the positrons. The positrons cool through cyclotron radiation with a characteristic time of $4 \mathrm{~s}$ (at a magnetic field of $1 \mathrm{~T}$ ), and sympathetically cool the ions (Fig. 2(b)) over a time interval of $\sim 80 \mathrm{~s}$, resulting in both ions and positrons accumulating around the potential well minimum. Positrons are then removed by applying a fast voltage pulse to one of the confining electrodes, thereby briefly opening the trap. The fastmoving positrons escape, while the slow-moving ions do not (Fig. 2(c)). The confining well is then modified to a predefined anharmonic shape and, after a short wait, the remaining ions are subjected to a voltage chirp drive applied to a neighboring electrode (Figs. 2(e) and 2(f)).

When a suitably tailored chirp is used, the axial motion of some of the confined ions phase-locks to the drive. As the chirp sweeps down in frequency, the energy of these autoresonantly driven ions increases until they are able to escape the well. These ions then follow the magnetic field lines onto an $\mathrm{MCP},{ }^{19}$ where charge is amplified through electron cascading upon impact (Fig. 1(c)). Electrons emitted from the back of the MCP are deposited on a charge collection plate and the resulting voltage signal is filtered, digitized, and stored in a computer. Once the signal has been stored, additional digital filtering is performed for further noise reduction, and charge information is recovered from the voltage signal by digitally deconvolving the collection plate system response. Since we record the timing between the escaped ions and the drive chirps, we can graph the frequencies at which ions escape. Note that time of flight $(\sim 1 \mu \mathrm{s})$ effects are negligible, as they occur at a time scale much shorter than the variations of charge at the collection plate $(\sim 1 \mathrm{~ms})$ typical of our measurements.

Different ion species have different steady-state bounce frequencies in the anharmonic well determined by their massto-charge ratio. Autoresonance forces particles of each particular species to follow the energy versus frequency paths shown in Fig. 3 and reach the top of the well (at $49.1 \mathrm{eV}$, measured with respect to the potential minimum at axial position 0; see Fig. 2(e)) at different drive frequencies. By analyzing

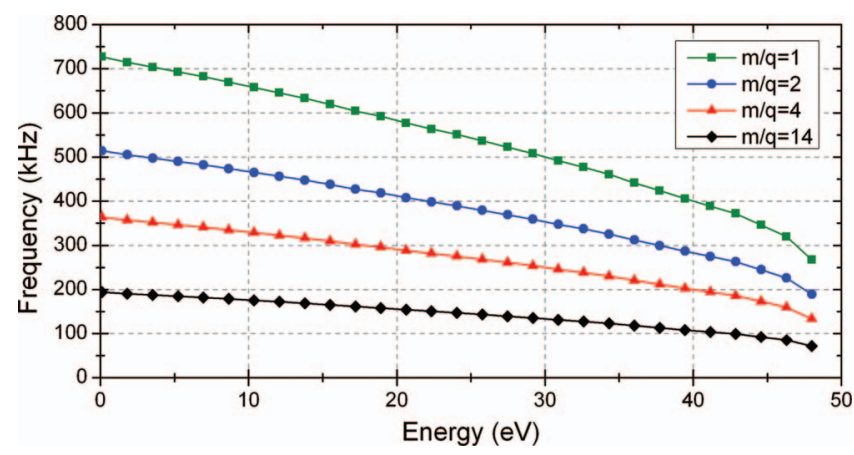

FIG. 3. Calculated bounce frequencies in the anharmonic potential well used for the experiments, of particles with mass-to-charge ratios 1 (green-squares), 2 (blue-circles), 4 (red-triangles), and 14 (black-diamonds), in units of proton mass/fundamental charge $\left(m_{p} / e\right)$. Here, and in what follows, the energy is chosen to be zero at the potential well minimum. the charge signal at the collection plate as a function of the drive frequency, it is possible to identify the different species in the well at the time of the chirp.

Images of ions arriving at the MCP taken with a CCD camera $^{19}$ (not shown) indicate charges concentrated within $\sim 1 \mathrm{~cm}$ of the trap axis. Since ions are expected to follow the field lines closely when extracted, and the magnetic field is $\sim 40$ times stronger inside the trap than at the location of the MCP, we can conclude that ions must be located within $\sim 1 \mathrm{~mm}$ of the axis of the trap during our experiments.

\section{SIMULATIONS}

A particle stepper code was written using a C-based Runge-Kutta method with adaptive step size control ${ }^{20}$ to simulate single-particle motion in the electrostatic potential well. Consistent with the experimental observations, it was assumed that the ions remained close to the trap axis at all times. Thus, the model reduced to the one dimensional (1D) motion of a charged particle in the anharmonic well of Fig. 2(e), plus an applied voltage chirp.

Given a value of mass-to-charge ratio of an ion " $m / q$ " (in units of $m_{p} / e$, where $m_{p}$ is the proton mass and $e$ is the fundamental charge) and specific initial conditions of energy and phase, the motion under the influence of the chirp was integrated. The escape time " $t$ " was measured, that is, the time when the particle left the potential well (if it left at all) after the start of the chirp, and the procedure was repeated over a wide range of possible values of initial conditions. Using these simulated data, normalized histograms of the number of escaping particles (with initial energy and phase in an experimentally expected range) versus escape time were created. Finally, the histograms were plotted against $(K / f)^{2}$, where $f$ was the frequency of the chirp at time " $t$ " and $K$ was an appropriately chosen constant (Fig. 4).

The choice $K=320 \mathrm{kHz}$ normalized the horizontal axis such that $\mathrm{H}^{+}$escaped at $(K / f)^{2}=1$. Since bounce frequencies scale like $f \sim \sqrt{q / m}$ (true even for anharmonic potentials),

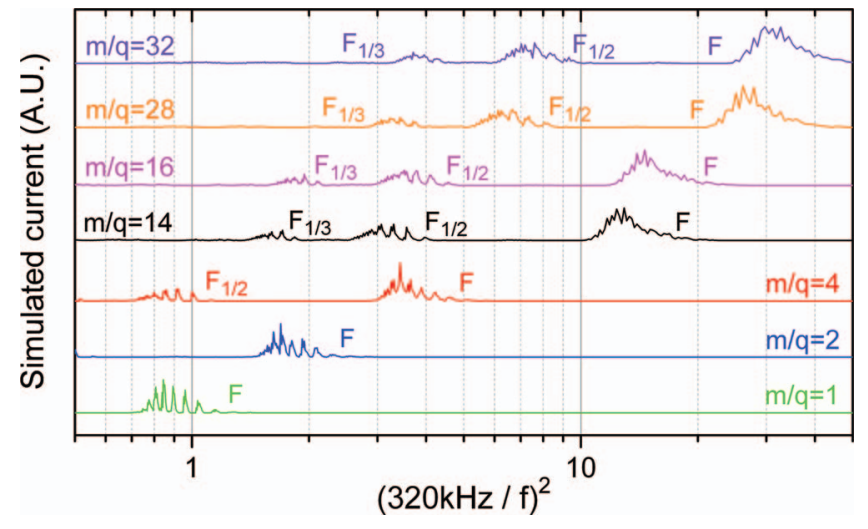

FIG. 4. Simulation histograms obtained for a $100 \mathrm{~ms}, 1 \mathrm{MHz}-10 \mathrm{kHz}, 4 \mathrm{~V}$ linear chirp applied to particles with $m / q=1,2,4,14,16,28$, and 32 and initial energies distributed uniformly in $0-49.1 \mathrm{eV}$. This energy range includes all possible initial energies and shows the full complexity of the particle dynamics in this nonlinear system. The peaks marked with an "F" correspond to particles driven out by the fundamental of the chirp, while " $F_{1 / 2}$ " and " $F_{1 / 3}$ " mark the first and second subharmonics. 
the $(K / f)^{2}$ mapping scales the escape locations of different species linearly with their mass-to-charge ratio. It also allows for plots with features that are independent of the length and functional form of the chirp, although only linear chirps were used for the work presented here.

The simulations show that ions with enough initial energy can be prematurely carried out of the potential well by subharmonics of the chirp drive. ${ }^{13}$ This effect is responsible for the appearance of additional peaks in the simulation histograms in locations of $(320 \mathrm{kHz} / f)^{2}$ far in numerical value from $m / q$. Care must therefore be taken in the analysis to distinguish peaks due to fundamental frequencies of the sweeps from subharmonic-produced ones.

Note that we have restricted the simulations to plausible species, namely $\mathrm{H}^{+}(m / q=1) ; \mathrm{H}_{2}^{+}$and $\mathrm{He}^{++}(m / q=2) ; \mathrm{He}^{+}$ $(m / q=4) ; \mathrm{N}^{+}$and $\mathrm{N}_{2}^{++}(m / q=14) ; \mathrm{O}^{+}$and $\mathrm{O}_{2}^{++}(m / q=16)$; $\mathrm{N}_{2}^{+}(m / q=28)$; and $\mathrm{O}_{2}^{+}(m / q=32)$. The fact that we search $\mathrm{N}^{+}$and $\mathrm{O}^{+}$is due to the possibility of positron-induced dissociation of the $\mathrm{N}_{2}$ and $\mathrm{O}_{2}$ molecules in the range of energies considered. ${ }^{16-18}$

Finally, note that attenuation of the chirp due to high pass filtering at the electrodes (used to shield the experiment from noise and decouple the chirp drive from the electrode bias voltages) becomes important at around $(320 \mathrm{kHz} / 100 \mathrm{kHz})^{2}$ $=10.2$. Thus, it is expected that peaks at locations larger than 10.2 will be increasingly difficult to observe in the experimental traces.

\section{TESTS AND ANALYSIS}

The first set of measurements involved changing the start frequency of the chirps while keeping the sweep rate fixed. As the calculated ion bounce frequencies for our anharmonic potential well show (Fig. 3), light particles cannot phase lock to chirps with low start frequencies, and consequently, the peak structure of the traces should vary for different choices of start frequencies. Note that ions escape due to subharmonics of the chirp at frequencies that are integer multiples of the escape (maximum energy) frequencies shown in Fig. 3. Thus, if for one particular species one chooses a sufficiently low start frequency and sweeps downwards (as is always the case), the subharmonics of the chirp will not excite particles of that species.

Figure 5 shows the experimental traces obtained for different choices of start frequencies, and compares them to simulations made for ions with initial energies in the range 0 $1.5 \mathrm{eV}$, consistent with separate experimental measurements of the initial ion energies when the trap is at $9 \mathrm{~K}$. The plots show that the simulations capture the main features of the dynamical system and are in good agreement with the experiments. They also allow an interpretation of the charge peaks. The group of peaks labeled "A" (in Fig. 5), for example, is consistent with the presence of $\mathrm{He}^{+}$. Although the simulations show that these peaks could also indicate the presence of $\mathrm{H}^{+}$, the fact that there are at most very small peaks at $(320 \mathrm{kHz} / f)^{2}$ $=2$ suggests that there is no $\mathrm{H}_{2}$ in the residual gas, and therefore, no normal source ${ }^{21}$ of $\mathrm{H}^{+}$. Group B is also consistent with $\mathrm{He}^{+}$, although it may have traces of $\mathrm{N}^{+}, \mathrm{N}_{2}^{++}, \mathrm{O}^{+}$, or
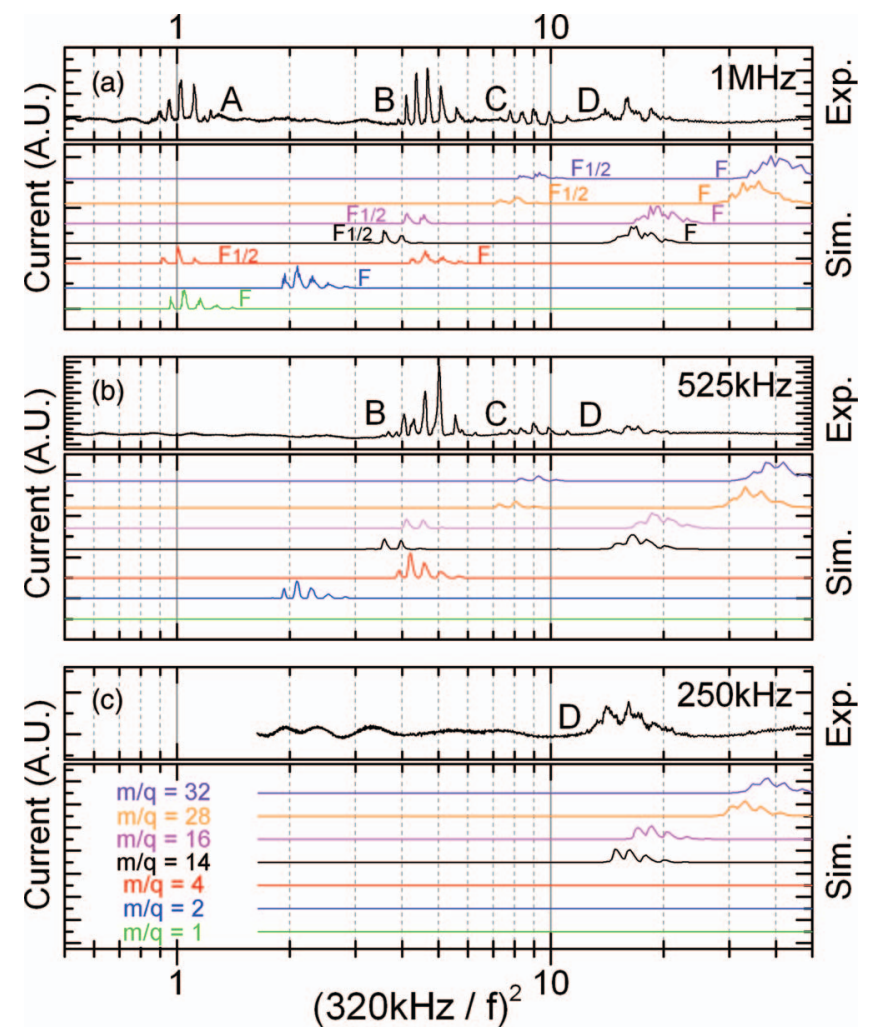

FIG. 5. (a) Experimental trace obtained with a $5 \mathrm{~V}, 100 \mathrm{~ms}, 1 \mathrm{MHz}-10 \mathrm{kHz}$ linear chirp (top), and simulation histograms made with a $4 \mathrm{~V}$, but otherwise similar, chirp (bottom). The $\sim 20 \%$ difference in drive amplitudes gives a better fit of the data, and is consistent with the uncertainty in our knowledge of the coupling between the drive electronics and the electrode. Unlike Fig. 4, this plot only includes initial energies in the $0-1.5 \mathrm{eV}$ range. (b) Experimental data for a $50 \mathrm{~ms}, 525 \mathrm{kHz}-10 \mathrm{kHz}$ linear chirp compared to the simulations. (c) Data and simulations for a linear $25 \mathrm{~ms}, 250 \mathrm{kHz}-10 \mathrm{kHz}$ chirp. See text for explanation of labels A, B, C, D.

$\mathrm{O}_{2}^{++}$. The disappearance of peaks in Figs. 5(b) and 5(c) reinforces this conclusion and is consistent with what is expected from Fig. 3. Group C is consistent with $\mathrm{N}_{2}^{+}$or $\mathrm{O}_{2}^{+}$at the first subharmonic. Group D is consistent with $\mathrm{N}^{+}$, although (to a lesser extent) it is also compatible with $\mathrm{O}^{+}$. This suggests that $\mathrm{N}_{2}$ and possibly $\mathrm{O}_{2}$ are present in the apparatus.

A further set of measurements involved changing the settings of nitrogen gas (which is used as a buffer gas to facilitate positron capture) pressures in the positron accumulator. When positrons are transferred into the main apparatus (Fig. 1(a)), some $\mathrm{N}_{2}$ will also necessarily enter. Consequently, higher $\mathrm{N}_{2}$ pressures in the accumulator should lead to higher $\mathrm{N}_{2}$ pressures in the mass spectrometer region.

Higher concentrations of $\mathrm{N}_{2}$ can have an effect on the energy distribution of the ions (for example, if the fraction of ions compared to positrons is significant, sympathetic cooling will no longer be as effective), and this in turn has an impact on the locations of the charge peaks. The simulations show that, in general, higher ion energies translate into charges escaping slightly earlier in time (i.e., at slightly higher chirp frequencies), and consequently, peaks appearing at lower values of $(320 \mathrm{kHz} / f)^{2}$.

Figure 6(a) shows experimental data taken at different accumulator pressures. The most striking difference is the ap- 


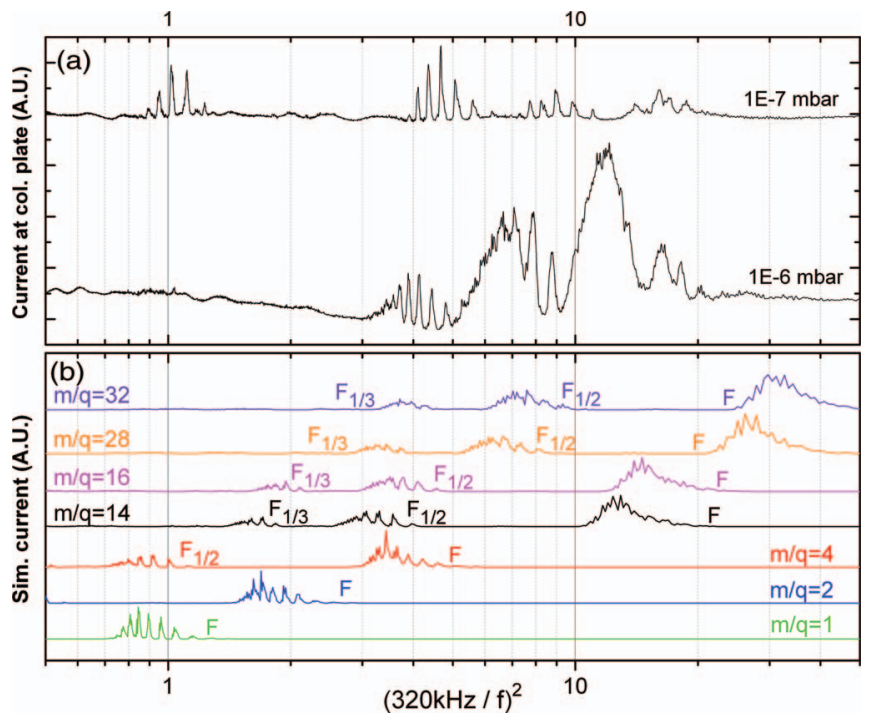

FIG. 6. (a) Experimental traces for different accumulator gas pressures when a $5 \mathrm{~V}$ linear, $1 \mathrm{MHz}-10 \mathrm{kHz}, 100 \mathrm{~ms}$ chirp is used $\left(1 \times 10^{-7} \mathrm{mbar}\right.$ is the default setting). (b) Simulations for ions with initial energies uniformly distributed between 0 and $49.1 \mathrm{eV}$, and a chirp amplitude of $4 \mathrm{~V}$.

pearance of pronounced peaks at $(320 \mathrm{kHz} / f)^{2}=7 \pm 1.0$ HWHM and $(320 \mathrm{kHz} / f)^{2}=12 \pm 1.5$ HWHM. Figure 6(b) shows the simulation results for ions with initial energies uniformly distributed in the wider range $0-49.1 \mathrm{eV}$. The simulation traces for $\mathrm{N}_{2}^{+}(m / q=28)$ and $\mathrm{N}^{+}, \mathrm{N}_{2}^{++}(m / q=14)$ provide a possible explanation for the appearance of the experimental charge peaks at $(320 \mathrm{kHz} / f)^{2}=7,12$, that is consistent with the expectation that nitrogen should be more clearly seen in the higher pressure trace. The fact that the simulated peaks at $(320 \mathrm{kHz} / f)^{2}=28$ are not clearly seen experimentally does not contradict this observation because, as stated previously, the chirp is heavily attenuated by the electrode filters at such low frequencies.

Finally, measurements were performed during a cool down of the apparatus. During this procedure, which lasts $\sim 5 \mathrm{~h}$, the temperature of the interior of the trap is brought down from $\sim 100 \mathrm{~K}$ to $9 \mathrm{~K}$, as measured by the Lakeshore Cernox sensor closest to the trapping region (attached to an electrode located $\sim 30 \mathrm{~cm}$ from the center of the trap). The change in temperature produces changes in the residual gas pressure and composition due to cryo-pumping on the walls ${ }^{6}$ that should lead to changes in the experimental traces. The changing pressure and density, and the consequent change in the collision frequencies of the particles, may also have an effect on ion formation, and ion-ion and ion-neutral reactions, ${ }^{22}$ which in turn affect the detected species and the relative strength of their signals. Also, as discussed above, the changing conditions may affect the energy distribution of the ions, which further complicates the analysis and comparison with the simulations.

Figures 7(b)-7(e) are experimental traces obtained $48 \mathrm{~min}, 72 \mathrm{~min}, 102 \mathrm{~min}$, and $198 \mathrm{~min}$ after the start of cool down that show the evolution of the vacuum in the trap. Based on the discussion above, the peaks at $(320 \mathrm{kHz} / f)^{2}=1$ are consistent with $\mathrm{H}^{+}$and/or $\mathrm{He}^{+}$at the first subharmonic (notice the changing peak structure around $\left.(320 \mathrm{kHz} / f)^{2}=1\right)$,

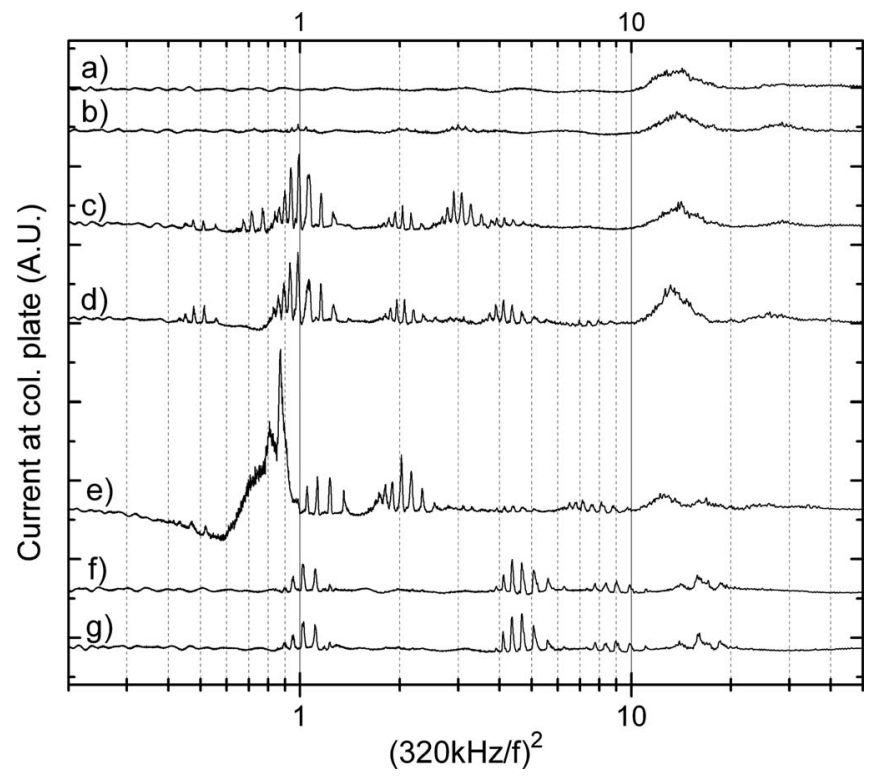

FIG. 7. Experimental traces obtained at different times during a cool down of the apparatus. Data were taken sequentially from start to end in the order (a) through (g). A linear $5 \mathrm{~V}, 1 \mathrm{MHz}-10 \mathrm{kHz}, 100 \mathrm{~ms}$ chirp was used for all measurements. See text for details of labeling.

and the groups at $(320 \mathrm{kHz} / f)^{2}=4,8,12$ are consistent with $\mathrm{He}^{+}, \mathrm{N}_{2}^{+}$, and/or $\mathrm{O}_{2}^{+}$, and $\mathrm{N}^{+}$and/or $\mathrm{O}^{+}$, respectively. The simulations show that the peaks seen at $(320 \mathrm{kHz} / f)^{2}=0.5$, 2 are consistent with $\mathrm{H}_{2}^{+}$, while the peaks at $(320 \mathrm{kHz} / f)^{2}$ $=3$ may indicate the presence of $\mathrm{H}_{3}^{+}$, which is allowed by the significant cross-section of the reaction $\mathrm{H}_{2}+\mathrm{H}_{2}^{+} \rightarrow \mathrm{H}_{3}^{+}$ $+\mathrm{H}$ at low energies. ${ }^{22}$ Figures 7(b)-7(d) also show a small peak close to $(320 \mathrm{kHz} / f)^{2}=30$ that is consistent with $\mathrm{N}_{2}^{+}$and possibly $\mathrm{O}_{2}^{+}$.

Figures 7(f) and 7(g) show the final (stable) mass spectra, corresponding to when the apparatus is cold at $9 \mathrm{~K}$. The most striking feature is the disappearance of the signals at $(320 \mathrm{kHz} / f)^{2}=0.5,2$, and 3 , which supports the conclusion that hydrogen is indeed present at early stages of cool down but is effectively pumped out by the end of the procedure.

Finally, Fig. 7(a) shows data taken at the start of cool down. In this case, the most visible peak is the one at $(320 \mathrm{kHz} / f)^{2}=12$, which suggests the presence of $\mathrm{N}^{+}, \mathrm{O}^{+}$, $\mathrm{N}_{2}^{+}$, and/or $\mathrm{O}_{2}^{+}$. Although not fully characterized, this is consistent with the observations made with higher accumulator pressures discussed above. There is also a small signal around $(320 \mathrm{kHz} / f)^{2}=30$ that was not visible in any of the previous experimental traces. It agrees with the simulations of $\mathrm{N}_{2}^{+}$ and/or $\mathrm{O}_{2}^{+}$, and suggests the presence of a large number of heavy gas molecules when the temperature of the apparatus is close to $100 \mathrm{~K}$.

We also ionized the residual gas through collisions with electrons instead of positrons. It was observed experimentally that, under stable (cold) conditions, the number of ions so produced correlated well with antiproton lifetime measurements and provided a way to estimate the density of the residual gas in the apparatus. ${ }^{3}$ Nevertheless, since electrons and ions have opposite charge, they tend to cool into separate potential wells, whereupon they lose contact and no further 
sympathetic cooling can occur. The very large energy spread of the ions made the experimental data difficult to analyze. Ionization with positrons avoids such problems.

\section{CONCLUSIONS}

This article describes the autoresonant spectroscopic determination of the residual gas in a charged particle trap at cryogenic temperatures and the tools developed for the analysis of the experimental data. It shows that the simulations for different drive frequency chirps give a good description of the experimental traces, allowing a reasonable interpretation to our measurements.

The simulations suggest that $\mathrm{He}, \mathrm{N}_{2}$, and possibly $\mathrm{O}_{2}$ are present when the trap is at $9 \mathrm{~K}$. Helium is not a common contaminant, so its presence may be an indication of small leaks from the surrounding liquid helium reservoir. As neither gaseous nitrogen nor oxygen can exist in thermodynamic equilibrium in a closed chamber at this temperature, their presence suggests that they originate from other parts of the apparatus. Nitrogen accompanying the transfer of positrons from the positron accumulator is the likely source of nitrogen. Other data taken when the trap is cooling down from $100 \mathrm{~K}$ show the gradual disappearance of other species, notably hydrogen.

The analysis is complicated by subharmonic resonances and the lack of precise knowledge of the initial energy distribution of the ions in the anharmonic well when the apparatus is not in stable (cold) conditions. Precise measurements of these energies would allow for more complete simulations of the traces during cool down and with higher positron accumulator pressures.

\section{ACKNOWLEDGMENTS}

We are grateful to CERN and the AD team. This work was supported by CNPq, FINEP/RENAFAE (Brazil); ISF (Israel); FNU (Denmark); VR (Sweden); NSERC, NRC/TRIUMF, AITF, FQRNT (Canada); DOE, NSF (USA); and EPSRC, the Royal Society, and the Leverhulme Trust (United Kingdom).

${ }^{1}$ J. S. deGrassie and J. H. Malmberg, "Waves and transport in the pure electron plasma," Phys. Fluids 23, 63 (1980).
${ }^{2}$ F. Anderegg, X.-P. Huang, E. Sarid, and C. F. Driscoll, "A new pure ion plasma device with laser induced fluorescence diagnostic," Rev. Sci. Instrum. 68, 2367 (1997).

${ }^{3} \mathrm{G}$. B. Andresen et al. (ALPHA collaboration), "Confinement of antihydrogen for 1000 seconds," Nat. Phys. 7, 558-564 (2011).

${ }^{4}$ G. Gabrielse, X. Fei, L. A. Orozco, R. L. Tjoelker, J. Haas, H. Kalinowsky, T. A. Trainor, and W. Kells, "Thousand-fold improvement in the measured antiproton mass," Phys. Rev. Lett. 65, 1317-1320 (1990).

${ }^{5} \mathrm{G}$. B. Andresen et al. (ALPHA collaboration), "Antimatter plasmas in a multipole trap for antihydrogen," Phys. Rev. Lett. 98, 023402 (2007).

${ }^{6} \mathrm{G}$. Lewin, Fundamentals of Vacuum Science and Technology (McGrawHill, 1965), p. 51-55.

${ }^{7}$ A. V. Ermakov and B. J. Hinch, "An electrostatic autoresonant ion trap mass spectrometer," Rev. Sci. Instrum. 81, 013107 (2010).

${ }^{8}$ G. Brucker and J. Rathbone, "Autoresonant trap mass spectrometry (ARTMS) for remote sensing applications," Int. J. Mass Spectrom. 295, 133-137 (2010).

${ }^{9}$ J. Fajans and L. Friedland, "Autoresonant (non stationary) excitation of a pendulum, Plutinos, plasmas and other nonlinear oscillators," Am. J. Phys. 69, 1096 (2001).

${ }^{10}$ J. Fajans, E. Gilson, and L. Friedland, "Autoresonant (nonstationary) excitation of the diocotron mode in non-neutral plasmas," Phys. Rev. Lett. 82, 4444-4447 (1999).

${ }^{11} \mathrm{G}$. B. Andresen et al. (ALPHA collaboration), "Autoresonant excitation of antiproton plasmas," Phys. Rev. Lett. 106, 025002 (2011).

${ }^{12}$ K. W. Murch, R. Vijay, I. Barth, O. Naaman, J. Aumentado, L. Friedland, and I. Siddiqi, "Quantum fluctuations in the chirped pendulum," Nat. Phys. 7, 105-108 (2011).

${ }^{13}$ J. Fajans, E. Gilson, and L. Friedland, "Second harmonic autoresonant control of the $1=1$ diocotron mode in pure electon plasmas," Phys. Rev. E 62, 4131 (2000)

${ }^{14}$ T. J. Murphy and C. M. Surko, "Positron trapping in an electrostatic well by inelastic collisions with nitrogen molecules," Phys. Rev. A 46, 5696 (1992).

${ }^{15} \mathrm{~L}$. V. Jørgensen et al. (ATHENA collaboration), "New source of dense, cryogenic positron plasmas," Phys. Rev. Lett. 95, 025002 (2005).

${ }^{16}$ H. Knudsen, L. Brun-Nielsen, M. Charlton, and M. R. Poulsen, "Single ionization of $\mathrm{H}_{2}, \mathrm{He}, \mathrm{Ne}$ and $\mathrm{Ar}$ by positron impact," J. Phys. B 23, 3955 (1990).

${ }^{17}$ H. Bluhme, "Ionisation by positron impact," Ph.D. dissertation (Institute of Physics and Astronomy, University of Aarhus, Denmark, 2000).

${ }^{18}$ J. P. Marler and C. M. Surko, "Positron-impact ionization, positronium formation, and electronic excitation cross sections for diatomic molecules," Phys. Rev. A 72, 062713 (2005).

${ }^{19} \mathrm{G}$. B. Andresen et al. (ALPHA collaboration), "Antiproton, positron, and electron imaging with a microchannel plate/phosphor detector," Rev. Sci. Instrum. 80, 123701 (2009).

${ }^{20}$ W. H. Press, S. A. Teukolsky, W. T. Vetterling, and B. P. Flannery, Numerical Recipes in C, 2nd ed. (Cambridge University Press, Cambridge, 1992), pp. 710-722.

${ }^{21} \mathrm{U}$. Bischler and E. Bertel, "Simple source of atomic hydrogen for ultrahigh vacuum applications," J. Vac. Sci. Technol. A 11, 458 (1993).

${ }^{22}$ A. V. Phelps, "Cross sections and swarm coefficients for $\mathrm{H}^{+}, \mathrm{H}_{2}^{+}, \mathrm{H}_{3}^{+}, \mathrm{H}$, $\mathrm{H}_{2}$ and $\mathrm{H}^{-}$in $\mathrm{H}_{2}$ for energies from $0.1 \mathrm{eV}$ to $10 \mathrm{keV}$," J. Phys. Chem. Ref. Data 19, 653 (1990). 